\title{
Detection of Tumor Multifocality Is Important for Prediction of Tumor Recurrence in Papillary Thyroid Microcarcinoma: A Retrospective Study and Meta-Analysis
}

\author{
Jung-Soo Pyo $\cdot$ Jin Hee Sohn \\ Guhyun Kang ${ }^{1}$
}

Department of Pathology, Kangbuk Samsung Hospital, Sungkyunkwan University School of Medicine, Seoul; 'Department of Pathology, Inje University Sanggye Paik Hospital, Inje University College of Medicine, Seoul, Korea

Received: March 3, 2016

Revised: March 29, 2016

Accepted: March 29, 2016

\section{Corresponding Author}

Jin Hee Sohn, MD

Department of Pathology, Kangbuk Samsung Hospital, Sungkyunkwan University School of

Medicine, 29 Saemunan-ro, Jongno-gu, Seoul 03181, Korea

Tel: +82-2-2001-2391

Fax: +82-2-2001-2398

E-mail: jhpath.sohn@samsung.com

\begin{abstract}
Background: The clinicopathological characteristics and conclusive treatment modality for multifocal papillary thyroid microcarcinoma (mPTMC) have not been fully established. Methods: A retrospective study, systematic review, and meta-analysis were conducted to elucidate the clinicopathological significance of mPTMC. We investigated the multiplicity of 383 classical papillary thyroid microcarcinomas (PTMCs) and the clinicopathological significance of incidental mPTMCs. Correlation between tumor recurrence and multifocality in PTMCs was evaluated through a systematic review and meta-analysis. Results: Tumor multifocality was identified in 103 of 383 PTMCs (26.9\%). On linear regression analysis, primary tumor diameter was significantly correlated with tumor number $\left(R^{2}=0.014, p=.021\right)$ and supplemental tumor diameter $\left(R^{2}=0.117, p=.023\right)$. Of 103 mPTMCs, 61 (59.2\%) were non-incidental, with tumor detected on preoperative ultrasonography, and $42(40.8 \%)$ were diagnosed (incidental mPTMCs) on pathological examination. Lymph node metastasis and higher tumor stage were significantly correlated with tumor multifocality. However, there was no difference in nodal metastasis or tumor stage between incidental and non-incidental mPTMCs. On meta-analysis, tumor multifocality was significantly correlated with tumor recurrence in PTMCs (odds ratio, 2.002; 95\% confidence interval, 1.475 to $2.719, p<.001$ ). Conclusions: Our results show that tumor multifocality in PTMC, regardless of manner of detection, is significantly correlated with aggressive tumor behavior.
\end{abstract}

Key Words: Papillary thyroid microcarcinoma; Multifocal; Incidental; Retrospective studies; Metaanalysis
Papillary thyroid microcarcinoma (PTMC) measuring $1 \mathrm{~cm}$ or less in diameter is the most common variant of papillary thyroid carcinoma (PTC). The incidence of PTMC has recently been increasing in many countries. This increase is partially attributed to the broader availability of ultrasonography (US) and computed tomography diagnostic modalities and to more frequent health screening with increased awareness of this tumor type. ${ }^{1}$ If the primary contributor to increasing PTMC incidence is improved detection, then the proportion of late-stage and large tumors should be declining. However, the incidence of larger tumors is also increasing. ${ }^{2}$ This pattern of increasing incidence cannot be completely explained by increased detection or more frequent health screening. Furthermore, over-diagnosis and over-treatment of PTMCs are important issues that warrant more detailed evaluation. Although PTMCs have similar molecular characteristics to PTCs larger than $1 \mathrm{~cm}$ (overt PTC), ${ }^{3}$ PTMCs generally have a better outcome profile than overt PTCs. ${ }^{4,5}$ However, some
PTMCs demonstrate loco-regional and lymph node recurrence, ${ }^{4}$ and diagnostic delays can result in higher rates of distant metastasis. $^{6}$

Old age, lymph node metastasis, extrathyroidal extension, and multifocality or bilaterality are considered high risk factors of PTC. ${ }^{7,8}$ Although the American Joint Committee on Cancer (AJCC) tumor node metastasis (TNM) classification system recommends describing tumor multifocality, TNM stage is not influenced by multifocality. ${ }^{9}$ Furthermore, definite evaluation and treatment modalities have not been established for multifocality in PTMC. Based on American Thyroid Association (ATA) management guidelines, ${ }^{7}$ patients with overt PTC with high risk factors are recommended to undergo total thyroidectomy with lymph node dissection. ${ }^{7,10}$ However, no definitive treatment guidelines for PTMC with high-risk factors have been recommended. Hemithyroidectomy or subtotal thyroidectomy is generally considered adequate treatment for unifocal PTMC (uPT- 
$\mathrm{MC}$ ), while the extent of surgical resection for multifocal PTMC (mPTMC) remains controversial. ${ }^{11}$ If incidental mPTMC is considered, the true rate of mPTMC could be higher than previously reported. The clinicopathological significance of multifocality in PTMC is not well established, and distinct treatment guidelines should be considered.

In the present study, we retrospectively investigated the clinicopathological significance and multifocality of PTMC with regard to primary tumor size. To evaluate the clinicopathological significance of incidentally detected $\mathrm{MPTMC}$, the rate of incidental MPTMC and its characteristics were investigated and compared with those of non-incidental mPTMC. Recurrence of mPTMC was also evaluated through meta-analysis.

\section{MATERIALS AND METHODS}

\section{Patients}

We investigated 383 consecutive classical PTMC patients from the Department of Pathology, Kangbuk Samsung Hospital, Sungkyunkwan University School of Medicine (Seoul, Korea) from January 1, 2010 to December 31, 2011. The correlations between multifocality and clinicopathological characteristics including age, sex, tumor size, extrathyroidal extension, lymph node metastasis, pTNM stage, and US findings were evaluated by reviewing medical charts, pathology records, and glass slides and based on the seventh edition AJCC TNM classification system. ${ }^{9}$ Patients had undergone either total thyroidectomy ( $\mathrm{n}=$ 237 ) or lobectomy (including hemithyroidectomy) $(\mathrm{n}=146)$ with lymph node dissection. The decisions to perform lymph node dissection and the extent of dissection were made by the surgeon based on ATA management guidelines ${ }^{7}$ and various risk-evaluation systems. ${ }^{5,9,12,13}$ The mean age of patients was $45.4 \pm 10.4$ years, the mean tumor size of the largest dominant tumor was $0.65 \pm 0.20 \mathrm{~cm}$, and the sex ratio (male:female) was $1: 3.85$. By AJCC stage grouping, there were 295 cases in stage I, 0 cases in stage II, 86 cases in stage III, and two cases in stage IV. The study protocol was approved by the Institutional Review Board (IRB) of Kangbuk Samsung Hospital (IRB No. KBC12202), who confirmed informed consents.

\section{Definition and evaluation of tumor multifocality}

Sections were cut to a thickness of $0.2 \mathrm{~cm}$ for postoperative pathological examination. All tumors detected during inspection were histologically examined. For evaluation of extrathyroidal extension, peritumoral parenchyma with thyroidal capsule was included. In addition, one section with normal-appearing parenchyma was included from each lobe. mPTMCs were defined as tumors with an intertumoral distance greater than 0.5 $\mathrm{cm}$ in the ipsilateral lobe or contralateral lobe of the primarytumor. ${ }^{14}$ The number of tumors ranged from two to six (mean, $2.36 \pm 0.70$ ), and we designated PTMCs other than the largest tumor as supplemental tumors. Cases were subdivided into three groups based on preoperative US findings. The first group included suspicious mPTMCs, the second group included uPTMCs accompanied by benign or indeterminate nodules, and the third group included suspicious uPTMCs. Within the second and third groups, incidental mPTMCs were defined as those discovered on pathological examination, which were not detected on preoperative US. US evaluation was performed by radiologists at our institution, and results were reported according to standard criteria.

\section{Literature search and selection criteria}

Relevant articles were obtained by searching PubMed and MEDLINE databases up to January 15, 2015. Searches were performed using the keywords "papillary thyroid carcinoma" and "multifocal." The title and abstract of all searched articles were screened for exclusion. Review articles were also screened to find additional eligible studies. Search results were then scanned according to the following inclusion and exclusion criteria: (1) PTC investigated in human tissue, (2) available information regarding tumor recurrence in uPTMC and MPTMC, (3) case reports or non-original articles were excluded, and (4) non-English language publications were excluded.

\section{Data extraction}

Data from all eligible studies were extracted by two authors. The following data were extracted from each of the eligible studies: the first author's name, year of publication, number of patients analyzed, and number of patients with tumor recurrence.

\section{Statistical analysis}

Statistical analyses were conducted using SPSS ver. 18.0 software (SPSS Inc., Chicago, IL, USA). The significance of tumor multifocality and correlations with clinicopathological parameters were determined by either chi-squre test or the Fisher exact test (two-sided). The relationship between tumor multifocality and tumor size was analyzed using a two-tailed Student's t test. Linear regression analysis was conducted to investigate correlations between primary tumor size and tumor multifocality, number of tumor, and supplemental tumor size. In addition, multivariate logistic regression analysis was performed to identify the 
most influential variables associated with tumor multifocality. To perform the meta-analysis, Comprehensive Meta-Analysis ver. 2 software (Biostat, Engelwood, NJ, USA) was used. Odds ratio (OR) with a $95 \%$ confidence interval (CI) was calculated by fixed-effect and random-effect models and used to evaluate the correlation between tumor multifocality and recurrence. Heterogeneity between studies was evaluated with the Q test, $\mathrm{I}^{2}$, and p-values. Publication bias was assessed via Begg's funnel plot and Egger's test. All statistical analysis was reviewed by a statistician. The results were two-sided and were considered statistically significant when $\mathrm{p}<.05$.

\section{RESULTS}

\section{Clinicopathological features of mPTMCs}

We initially investigated the correlations between tumor multifocality and clinicopathological parameters in 383 resected classical PTMCs. Tumor multifocality was noted in 103 of 383 PTMCs (26.9\%), and nodal metastasis occurred at significantly higher rates in mPTMCs than in uPTMCs ( $\mathrm{p}=.003)$. Patients who underwent total thyroidectomy showed a higher incidence of tumor multifocality than patients who underwent lobectomy $(\mathrm{p}<.001)$. For the 83 mPTMCs in which total thyroidectomy was undertaken, tumor bilaterality was $62.7 \%$ (52 of 83). mPTMC showed a significant correlation with higher
TNM stage than uPTMC $(\mathrm{p}<.001)$. There were no significant differences with respect to age, sex, tumor size, or extrathyroidal extension (Table 1). On multivariate analysis, tumor multifocality was significantly correlated with lymph node metastasis $(\mathrm{p}=$ .002 ) but not age ( $\geq 45$ years), sex, or extrathyroidal extension.

On preoperative US, tumor multiplicity was found in 180 cases including 54 suspicious mPTMC cases. Among suspicious mPTMCs, 41 cases were confirmed as mPTMCs (non-incidental mPTMCs) on pathological examination. For the remaining 126 suspicious uPTMCs accompanied by benign or indeterminate nodules, 20 cases were confirmed to be MPTMC (non-incidental mPTMC). Nineteen cases (three suspicious mPTMC cases and 16 non-suspicious mPTMC cases) involved incidentally found PTMCs (incidental mPTMCs) that were not identified preoperatively. In 203 cases regarded as uPTMCs on preoperative US, 23 supplemental PTMCs were incidentally discovered on pathological examination (incidental mPTMCs). In total, 103 mPTMCs (61 non-incidental and 42 incidental) of 383 PTMCs were identified (Fig. 1).

\section{Distribution of mPTMC based on primary tumor size}

The distribution of mPTMCs based on primary tumor size was investigated and is shown in Fig. 2A. The rate of mPTMC occurrence was $0 \%-34.4 \%$ and was shown to increase with increasing primary tumor size on linear regression analysis $\left(\mathrm{R}^{2}=\right.$

Table 1. Correlation between tumor multifocality and clinicopathological features in PTMCs

\begin{tabular}{|c|c|c|c|}
\hline Clinicopathological feature $(n=383)$ & Multifocal PTMC ( $n=103,26.9 \%)$ & Unifocal PTMC ( $n=280,73.1 \%)$ & p-value \\
\hline \multicolumn{4}{|l|}{ Age (yr) } \\
\hline$<45$ & $43(41.7)$ & $144(51.4)$ & .093 \\
\hline$\geq 45$ & $60(58.3)$ & $136(48.6)$ & \\
\hline \multicolumn{4}{|l|}{ Gender } \\
\hline Male & $18(17.5)$ & $61(21.8)$ & .355 \\
\hline Female & $85(82.5)$ & $219(78.2)$ & \\
\hline Total thyroidectomy & $83(80.6)$ & $154(55.0)$ & $<.001$ \\
\hline Lobectomy & $20(19.4)$ & $126(45.0)$ & \\
\hline \multicolumn{4}{|l|}{ Extrathyroidal extension } \\
\hline Present & $54(52.4)$ & $125(44.6)$ & .176 \\
\hline Absent & $49(47.6)$ & $155(55.4)$ & \\
\hline \multicolumn{4}{|l|}{ Lymph node metastasis } \\
\hline Absent & $51(49.5)$ & $185(66.1)$ & \\
\hline \multicolumn{4}{|l|}{ Tumor stage } \\
\hline I & $52(50.5)$ & $203(72.5)$ & $<.001$ \\
\hline$\|$ & 0 & 0 & \\
\hline III & $51(49.5)$ & 75 (26.8) & \\
\hline IV & 0 & $2(0.7)$ & \\
\hline
\end{tabular}

Values are presented as number (\%) or mean \pm standard deviation.

PTMC, papillary thyroid microcarcinoma. 


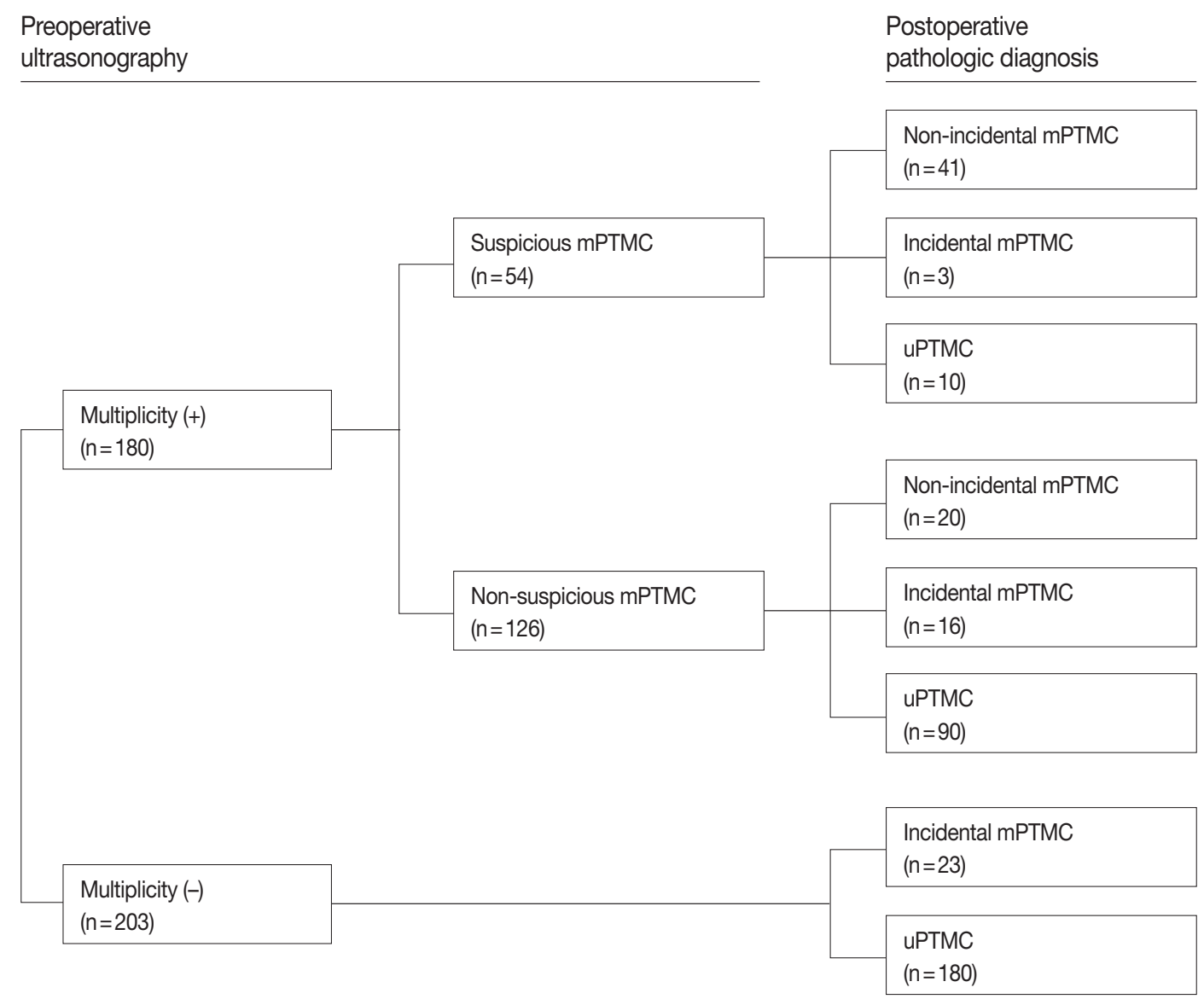

Fig. 1. Diagnostic flow in 383 patients based on preoperative ultrasonography. mPTMC, multifocal papillary thyroid microcarcinoma; UPTMC, unifocal papillary thyroid microcarcinoma.

0.519 and $\mathrm{p}=.019)$. PTMCs were divided with a cutoff of 0.3 $\mathrm{cm}$, and the MPTMC rate was $5.9 \%$ in PTMCs smaller than 0.3 $\mathrm{cm}$ and $27.9 \%$ in PTMCs larger than $0.3 \mathrm{~cm}$. In addition, the number of tumors and supplemental tumor size were significantly increased according to primary tumor size $\left(\mathrm{R}^{2}=0.014, \mathrm{p}=\right.$ .021 and $\mathrm{R}^{2}=0.117, \mathrm{p}=.023$, respectively) (Fig. $2 \mathrm{~B}, \mathrm{C}$ ).

\section{Lymph node metastasis in incidental and non-incidental mPTMC}

Forty-two cases (40.8\%) were incidentally diagnosed as mPT$\mathrm{MC}$ via postoperative pathological examination. To understand the significance of incidentally discovered supplemental tumors in MPTMC, we compared clinicopathological characteristics between incidental and non-incidental mPTMCs. The mean sizes of supplemental tumors were $0.23 \pm 0.12 \mathrm{~cm}$ in incidental mPTMCs and $0.41 \pm 0.17 \mathrm{~cm}$ in non-incidental mPTMCs. As expected, supplemental tumor size was significantly smaller in incidental mPTMCs than in non-incidental mPTMCs $(\mathrm{p}<.001)$, even though the largest tumor size did not differ $(\mathrm{p}=.870)$. The rate of extrathyroidal extension was higher in incidental mPTMCs than in non-incidental mPTMCs $(\mathrm{p}=.016)$, but there was no difference in lymph node metastasis $(\mathrm{p}=$.199) (Table 2).

\section{Systematic review and meta-analysis}

We performed a systematic review and meta-analysis to confirm the difference of tumor recurrence between UPTMC and mPTMC. One hundred fifty-six studies were identified through a database search and were screened (Fig. 3); 148 of these studies were excluded due to no or insufficient information (123), studies on other disease (10), case reports or non-original articles (13), and non-English-language articles (2). Eight eligible studies and 5,665 patients were included in the current meta-analysis. ${ }^{15-22}$ Tumor multifocality was found in 1,844 of 5,665 overall PTMCs (32.6\%). In the eligible studies, the rates of tumor multifocality were $24.0 \%-42.5 \%$. Our meta-analysis showed significant correlation between tumor recurrence and MPTMCs in fixed-effect (OR, 2.002; 95\% CI, 1.475 to 2.719; p<.001) and random-effect (OR, 2.118; 95\% CI, 1.323 to 3.390; $\mathrm{p}=.002)$ 

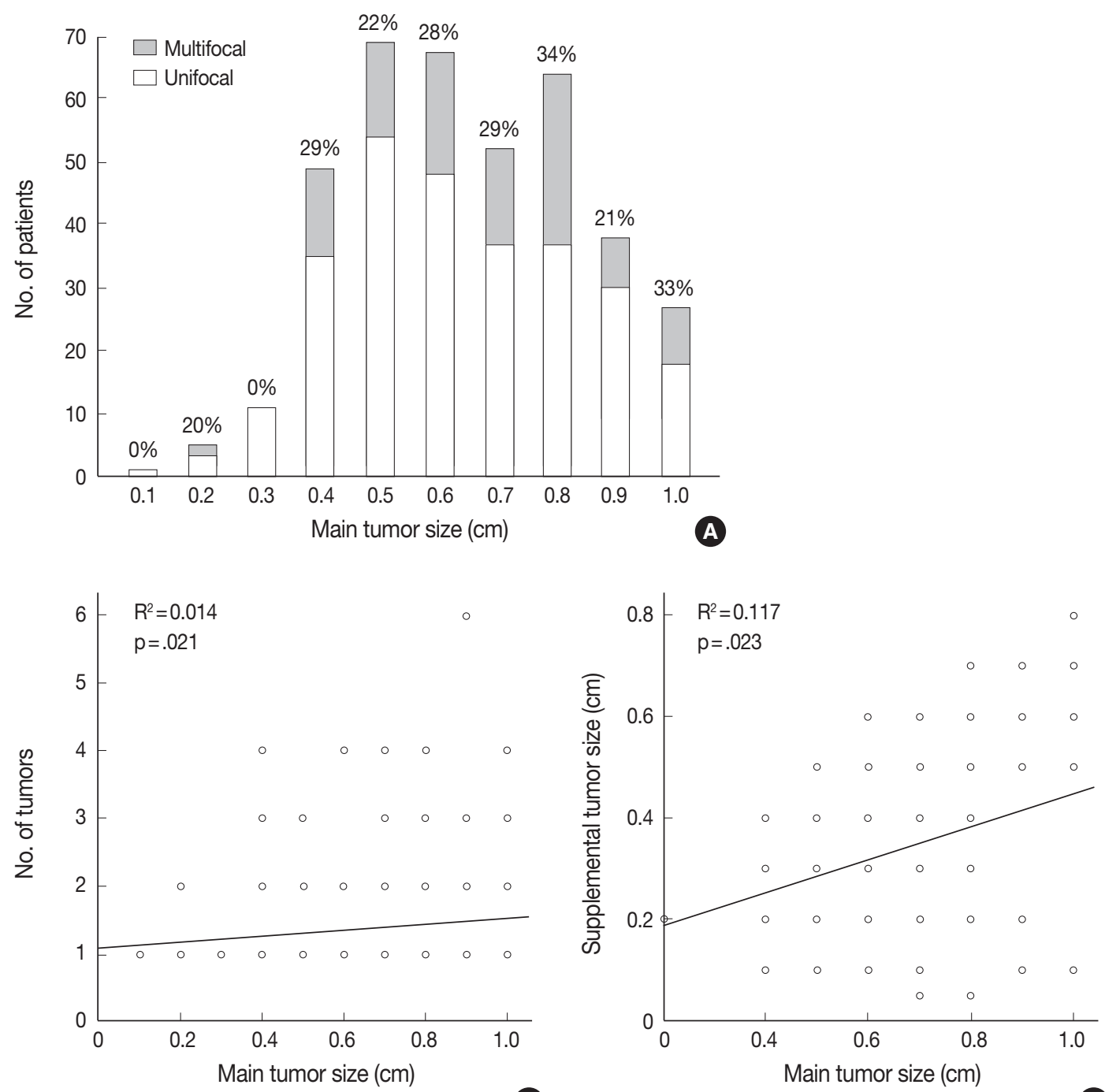

B

C

Fig. 2. Correlation between primary tumor size and multifocality in papillary thyroid microcarcinoma (PTMC). (A) Distribution of tumor multifocality based on primary tumor size in PTMC. (B) Correlation between primary tumor size and number of tumors using linear regression. (C) Correlation between primary and supplemental tumor size by linear regression.

models (Fig. 4). On sensitivity analysis, OR ranges were 1.736 2.277 and 1.907-2.417 in fixed-effect and random-effect models, respectively, and estimated ORs were not affected by eligible studies. No significant heterogeneity was identified $\left(\mathrm{I}^{2}=47.4 \%\right.$, $\mathrm{p}=.065)$. There was no definite asymmetry in Begg's funnel plot (data not shown). Egger's test showed no evidence of publication bias $(\mathrm{p}=.432)$.

\section{DISCUSSION}

Multifocal PTCs are associated with loco-regional recurrence and lymph node metastasis; ${ }^{10}$ however, the clinicopathological significance and appropriate treatment modalities for MPTMC remain unclear. In addition, little is known about the clinicopathological characteristics of incidental mPTMCs. To the best of our knowledge, this is the first report showing the clinicopathological significance of incidental mPTMCs and the correlation between tumor recurrence and mPTMCs using meta-analysis.

The incidence of PTMCs has rapidly increased in recent years. ${ }^{23-25}$ It is debated whether PTMC is a normal finding, equivalent to disease, or a precursor of overt PTC. ${ }^{23}$ Ito et al. ${ }^{4}$ reported that the rate of PTMC enlargement without unfavorable features was $6.4 \%$ at 5 years and $15.9 \%$ at 10 years. In previous reports, distant metastasis and mortality occurred more rarely in PTMC than in overt PTC. ${ }^{26,27}$ However, there are many reports showing that PTMCs show similar rates of extrathyroidal extension 
Table 2. Analysis of clinicopathological features between incidental and non-incidental mPTMCs

\begin{tabular}{|c|c|c|c|}
\hline \multirow{2}{*}{ Clinicopathological feature $(n=103)$} & \multicolumn{2}{|c|}{ mPTMCs } & \multirow{2}{*}{$\mathrm{p}$-value } \\
\hline & Incidental $(n=42,40.8 \%)$ & Non-incidental $(n=61,59.2 \%)$ & \\
\hline \multicolumn{4}{|l|}{ Age (yr) } \\
\hline$<45$ & $18(42.9)$ & $25(41.0)$ & .850 \\
\hline$\geq 45$ & $24(57.1)$ & $36(59.0)$ & \\
\hline \multicolumn{4}{|l|}{ Gender } \\
\hline Male & $5(11.9)$ & $13(21.3)$ & .217 \\
\hline Female & $37(88.1)$ & 48 (78.7) & \\
\hline Main tumor size (cm) & $0.66 \pm 0.19$ & $0.67 \pm 0.19$ & .801 \\
\hline Supplemental tumor size (cm) & $0.23 \pm 0.12$ & $0.41 \pm 0.17$ & $<.001$ \\
\hline Total thyroidectomy & $29(69.0)$ & $54(88.5)$ & .014 \\
\hline Lobectomy & $13(31.0)$ & $7(11.5)$ & \\
\hline \multicolumn{4}{|l|}{ Extrathyroidal extension } \\
\hline Present & $28(66.7)$ & $26(42.6)$ & .016 \\
\hline Absent & $14(33.3)$ & $35(57.4)$ & \\
\hline \multicolumn{4}{|l|}{ Lymph node metastasis } \\
\hline Present & $18(42.9)$ & $34(55.7)$ & .199 \\
\hline Absent & $24(57.1)$ & $27(44.3)$ & \\
\hline \multicolumn{4}{|l|}{ Tumor stage } \\
\hline I & $21(50.0)$ & 30 (49.2) & $>.999$ \\
\hline$\|$ & 0 & 0 & \\
\hline III & $21(50.0)$ & 31 (50.8) & \\
\hline IV & 0 & 0 & \\
\hline
\end{tabular}

Values are presented as number (\%) or mean \pm standard deviation. mPTMC, multifocal papillary thyroid microcarcinoma.

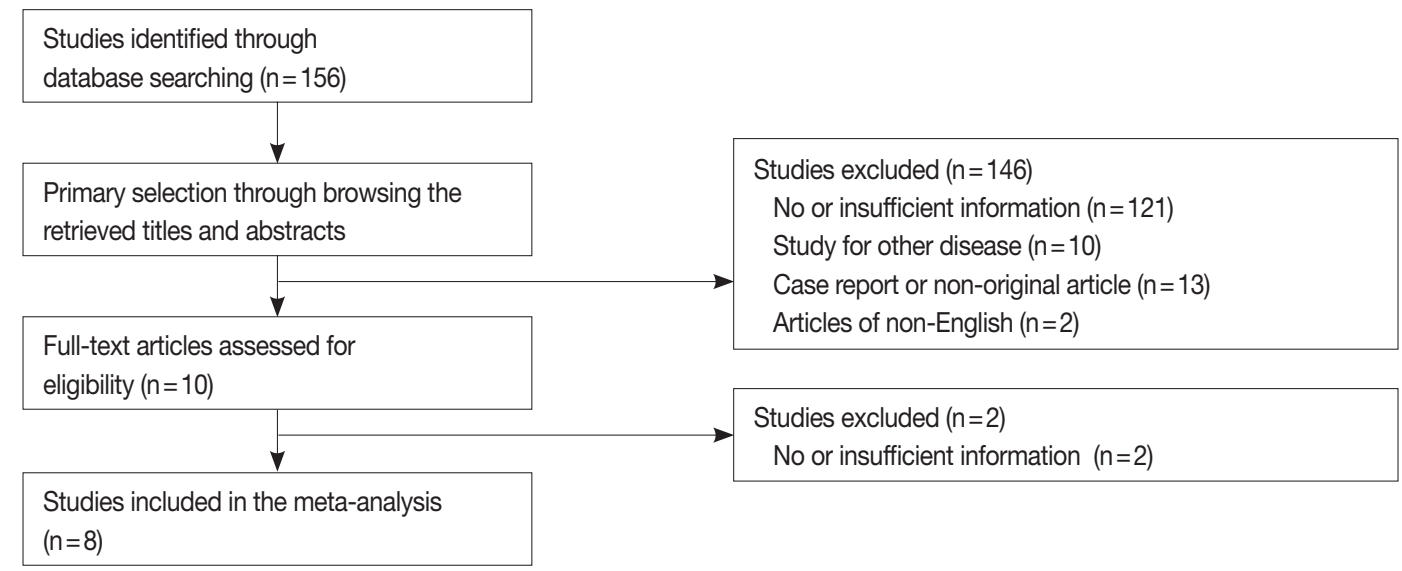

Fig. 3. Flow chart of study search and selection.

and nodal metastasis $(40.3 \%$ to $64.1 \%)$. $^{23-25,28,29}$ In addition, a recent meta-analysis showed that total thyroidectomy was significantly correlated with lower recurrence and mortality rates in PTMC. ${ }^{30}$ Patients who underwent total thyroidectomy showed a higher incidence of tumor multifocality than patients who underwent lobectomy ( $<$.001). In 83 mPTMCs that underwent total thyroidectomy, tumor bilaterality was $62.7 \%$ (52 of 83). Still, the unfavorable factors that can result in aggressive behavior and impact prognosis in PTMCs remain unclear.

Tumor multifocality is not considered a high-risk factor in systems including AGES (Age, Grade, Extent, Size), AMES (Age, Distant metastasis, Extent, Size), MACIS (Distant metastasis, Age, Completeness of resection, Local invasion, Size), and GAMES (Grade, Age, Distant metastasis, Extent, Size). ${ }^{6,12,13}$ Our data showed that tumor multifocality was significantly associated with nodal metastasis in $26.9 \%$ of total PTMCs, as in previous studies. ${ }^{15,17-19}$ However, in the PTMCs of some previous studies, the correlation between tumor multifocality and recurrence wascontroversial. ${ }^{15-22}$ Further cumulative prospective studies or meta-analysis should be performed to determine the clinico- 


\begin{tabular}{|c|c|c|c|c|c|}
\hline \multirow[t]{2}{*}{ Model } & \multirow[t]{2}{*}{ Study name } & \multicolumn{4}{|c|}{ Statistics for each study } \\
\hline & & Odds ratio & Lower limit & Upper limit & $\mathrm{p}$-value \\
\hline & Baudin $1998^{15}$ & 7.781 & 1.647 & 36.758 & .010 \\
\hline & Chow $2003^{16}$ & 3.375 & 1.028 & 11.085 & .045 \\
\hline & Hay $2008^{17}$ & 2.893 & 1.621 & 5.161 & .000 \\
\hline & $\operatorname{Kim} 2015^{18}$ & 1.838 & 0.948 & 3.565 & .072 \\
\hline & Lombardi $2010^{19}$ & 11.509 & 1.420 & 93.285 & .022 \\
\hline & Neuhold $2011^{20}$ & 0.733 & 0.076 & 7.084 & .788 \\
\hline & Ross $2009^{21}$ & 1.368 & 0.706 & 2.651 & .353 \\
\hline & Zheng $2013^{22}$ & 0.960 & 0.434 & 2.122 & .920 \\
\hline Fixed & & 2.002 & 1.475 & 2.719 & .000 \\
\hline Randon & & 2.118 & 1.323 & 3.390 & .002 \\
\hline
\end{tabular}

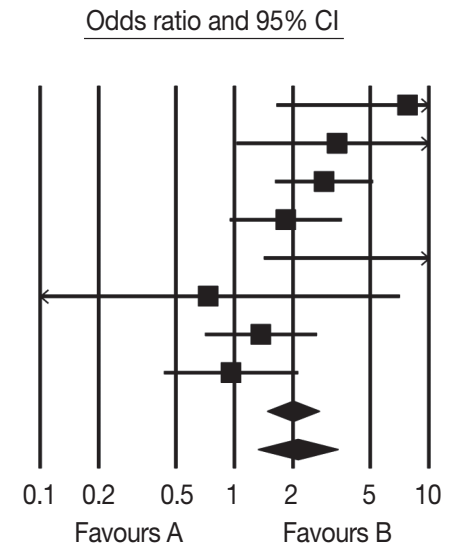

Fig. 4. Forest plot diagram of tumor recurrence difference between multifocal and unifocal papillary thyroid microcarcinomas. ${ }^{15-22} \mathrm{Cl}$, confidence interval.

pathological significance of mPTMC. Our meta-analysis showed that tumor recurrence was significantly higher in MPTMC than in uPTMC (Fig. 4). Regrettably, our data could not be included in the meta-analysis due to limitations including no recurrence cases during follow-up and short follow-up period (3 years). In addition, the current meta-analysis did not allow subgroup analysis based on positive or negative resection margins due to insufficient information provided in the eligible studies. Kuo $e t$ al. ${ }^{31}$ reported that mPTMC was associated with worse survival than UPTMC but reported no difference in survival between multifocal overt PTC (>1 cm) and mPTMC. Our results and earlier studies suggest that tumor multifocality in PTMCs, regardless of tumor size, is a useful predictive factor of aggressive tumor behavior, such as lymph node metastasis and tumor recurrence. It might be appropriate to manage patients with $\mathrm{mPT}$ MC differently than patients with uPTMC having no unfavorable factors.

Incidental PTMC is defined as PTMC incidentally discovered and confirmed by gross and/or microscopic examination from surgical specimens resected for the evaluation of other disease entities. ${ }^{32,33}$ However, no clear definition for incidental mPTMC has been previously provided. In this study, we defined incidental mPTMC as supplemental tumors not detected on preoperative US that were discovered on pathological examination. Although these supplemental tumors are important for diagnosis of multifocality, the characteristics of supplemental tumors have not been previously described. In the present study, incidental mPTMCs accounted for $40.8 \%$ of the total mPTMC according to our criteria. Interestingly, incidental mPTMC was not found in PTMCs $0.3 \mathrm{~cm}$ or smaller. However, the lower limit of tumor size detectable on US is unclear, and more controlled and careful histological examination for entirely submitted cases is need- ed to identify tumor multifocality. The mean size of incidentally discovered supplemental tumors was $0.27 \pm 0.15 \mathrm{~cm}$, which was significantly smaller than non-incidental tumors $(\mathrm{p}<.001)$. The supplemental tumor size of MPTMC decreased with decreasing primary tumor size (Fig. 2). The smaller size explains the lack of detection during preoperative US. However, there was no difference in nodal metastasis rate between incidental and non-incidental mPTMC. This finding suggests that tumor multifocality itself, rather than the manner of detection, is related to lymph node metastasis. In addition, the detection rate of incidental mPTMC might influence the rate of MPTMC. If these supplemental tumors are missed in pathological examination, cases are diagnosed as UPTMC and not mPTMC. Therefore, detailed examination for multiple tumors using preoperative US study and adequate postoperative pathological examination is essential in the diagnosis of PTMC.

We previously reported that evaluation of tumor multifocality based on total surface area is useful to distinguish aggressive mPTMC from less aggressive mPTMC and uPTMC. ${ }^{14}$ On that basis, we concluded that careful detection of multifocal tumors is important despite discrepant reporting on multifocality. The discrepancies largely seem to result from different manners of pathological examination and the number of sections analyzed. Although radiological tools have improved, tumors smaller than $0.3 \mathrm{~cm}$ are not easily detected via radiological examination. In the present study, only four of 383 PTMCs were smaller than $0.3 \mathrm{~cm}$. Among them, two cases were $0.2 \mathrm{~cm}$ on histological examination, after appearing larger than $0.3 \mathrm{~cm}$ on preoperative US. Suspicious nodules on preoperative US in the remaining two cases were confirmed as benign nodules on histological examination. Another two incidentally detected nodules $(0.2 \mathrm{~cm}$ and $0.1 \mathrm{~cm}$ ) were diagnosed as PTMC. Therefore, there were no sus- 
picious nodules smaller than $0.3 \mathrm{~cm}$ reported preoperatively. In addition, it is difficult to confirm PTMC in preoperative fineneedle aspiration for tumors smaller than $0.5 \mathrm{~cm},{ }^{34}$ which require thin sections in postoperative gross examination for non-detected small nodules. Because it is difficult to cut 0.2-cm-thick slices of specimens prior to fixation, thin sectioning after proper fixation is required for assessment of tumor multifocality.

In conclusion, this study demonstrates that tumor multifocality in PTMC is significantly correlated with lymph node metastasis. Our meta-analysis revealed a positive correlation between tumor multifocality and tumor recurrence in PTMC. Therefore, careful attention should be paid to detection of MPTMC, which behaves differently from uPTMC, on preoperative and postoperative examination.

\section{Conflicts of Interest}

No potential conflict of interest relevant to this article was reported.

\section{REFERENCES}

1. Mercante G, Frasoldati A, Pedroni C, et al. Prognostic factors affecting neck lymph node recurrence and distant metastasis in papillary microcarcinoma of the thyroid: results of a study in 445 patients. Thyroid 2009; 19: 707-16.

2. Enewold L, Zhu K, Ron E, et al. Rising thyroid cancer incidence in the United States by demographic and tumor characteristics, 19802005. Cancer Epidemiol Biomarkers Prev 2009; 18: 784-91.

3. Park YJ, Kim YA, Lee YJ, et al. Papillary microcarcinoma in comparison with larger papillary thyroid carcinoma in BRAFV600E mutation, clinicopathological features, and immunohistochemical findings. Head Neck 2010; 32: 38-45.

4. Ito $\mathrm{Y}$, Miyauchi A, Inoue $\mathrm{H}$, et al. An observational trial for papillary thyroid microcarcinoma in Japanese patients. World J Surg 2010; 34: 28-35.

5. Sugitani I, Toda K, Yamada K, Yamamoto N, Ikenaga M, Fujimoto Y. Three distinctly different kinds of papillary thyroid microcarcinoma should be recognized: our treatment strategies and outcomes. World J Surg 2010; 34: 1222-31.

6. Mazzaferri EL. Managing small thyroid cancers. JAMA 2006; 295: 2179-82.

7. American Thyroid Association (ATA) Guidelines Taskforce on Thyroid Nodules and Differentiated Thyroid Cancer, Cooper DS, Doherty GM, et al. Revised American Thyroid Association management guidelines for patients with thyroid nodules and differen- tiated thyroid cancer. Thyroid 2009; 19: 1167-214.

8. Lee E, Jung W, Woo JS, et al. Tumor sprouting in papillary thyroid carcinoma is correlated with lymph node metastasis and recurrence. Korean J Pathol 2014; 48: 117-25.

9. Edge SB, Byrd DR, Compton CC, Fritz AG, Greene FL, Trotti A. AJCC cancer staging manual. 7th ed. New York: Springer-Verlag, 2009; 87-96.

10. Mazeh H, Samet $Y$, Hochstein D, et al. Multifocality in well-differentiated thyroid carcinomas calls for total thyroidectomy. Am J Surg 2011; 201: 770-5.

11. Dietlein M, Luyken WA, Schicha H, Larena-Avellaneda A. Incidental multifocal papillary microcarcinomas of the thyroid: is subtotal thyroidectomy combined with radioiodine ablation enough? Nucl Med Commun 2005; 26: 3-8.

12. Rodríguez-Cuevas S, Labastida-Almendaro S, Cortés-Arroyo H, López-Garza J, Barroso-Bravo S. Multifactorial analysis of survival and recurrences in differentiated thyroid cancer: comparative evaluation of usefulness of AGES, MACIS, and risk group scores in Mexican population. J Exp Clin Cancer Res 2002; 21: 79-86.

13. Voutilainen PE, Siironen P, Franssila KO, Sivula A, Haapiainen RK, Haglund $\mathrm{CH}$. AMES, MACIS and TNM prognostic classifications in papillary thyroid carcinoma. Anticancer Res 2003; 23: 4283-8.

14. Pyo JS, Sohn JH, Kang G, Kim DH, Yun J. Total surface area is useful for differentiating between aggressive and favorable multifocal papillary thyroid carcinomas. Yonsei Med J 2015; 56: 355-61.

15. Baudin E, Travagli JP, Ropers J, et al. Microcarcinoma of the thyroid gland: the Gustave-Roussy Institute experience. Cancer 1998; 83: 553-9.

16. Chow SM, Law SC, Chan JK, Au SK, Yau S, Lau WH. Papillary microcarcinoma of the thyroid-Prognostic significance of lymph node metastasis and multifocality. Cancer 2003; 98: 31-40.

17. Hay ID, Hutchinson ME, Gonzalez-Losada T, et al. Papillary thyroid microcarcinoma: a study of 900 cases observed in a 60-year period. Surgery 2008; 144: 980-7.

18. Kim KJ, Kim SM, Lee YS, Chung WY, Chang HS, Park CS. Prognostic significance of tumor multifocality in papillary thyroid carcinoma and its relationship with primary tumor size: a retrospective study of 2,309 consecutive patients. Ann Surg Oncol 2015; 22: 125-31.

19. Lombardi CP, Bellantone R, De Crea C, et al. Papillary thyroid microcarcinoma: extrathyroidal extension, lymph node metastases, and risk factors for recurrence in a high prevalence of goiter area. World J Surg 2010; 34: 1214-21.

20. Neuhold N, Schultheis A, Hermann M, Krotla G, Koperek O, Birner P. Incidental papillary microcarcinoma of the thyroid: further evidence of a very low malignant potential: a retrospective clinicopathological study with up to 30 years of follow-up. Ann Surg Oncol 
2011; 18: 3430-6.

21. Ross DS, Litofsky D, Ain KB, et al. Recurrence after treatment of micropapillary thyroid cancer. Thyroid 2009; 19: 1043-8.

22. Zheng X, Wei S, Han Y, et al. Papillary microcarcinoma of the thyroid: clinical characteristics and BRAFV600E mutational status of 977 cases. Ann Surg Oncol 2013; 20: 2266-73.

23. Haymart MR, Cayo M, Chen H. Papillary thyroid microcarcinomas: big decisions for a small tumor. Ann Surg Oncol 2009; 16: 3132-9.

24. Noguchi S, Yamashita H, Uchino S, Watanabe S. Papillary microcarcinoma. World J Surg 2008; 32: 747-53.

25. Mazzaferri EL. Managing thyroid microcarcinomas. Yonsei Med J 2012; 53: 1-14.

26. Papini E, Guglielmi R, Bianchini A, et al. Risk of malignancy in nonpalpable thyroid nodules: predictive value of ultrasound and color-Doppler features. J Clin Endocrinol Metab 2002; 87: 1941-6.

27. Strate SM, Lee EL, Childers JH. Occult papillary carcinoma of the thyroid with distant metastases. Cancer 1984; 54: 1093-100.

28. Roh JL, Kim JM, Park CI. Central cervical nodal metastasis from papillary thyroid microcarcinoma: pattern and factors predictive of nodal metastasis. Ann Surg Oncol 2008; 15: 2482-6.

29. Cheema Y, Olson S, Elson D, Chen H. What is the biology and optimal treatment for papillary microcarcinoma of the thyroid? J Surg Res 2006; 134: 160-2.

30. Macedo FI, Mittal VK. Total thyroidectomy versus lobectomy as initial operation for small unilateral papillary thyroid carcinoma: a meta-analysis. Surg Oncol 2015; 24: 117-22.

31. Kuo SF, Lin SF, Chao TC, Hsueh C, Lin KJ, Lin JD. Prognosis of multifocal papillary thyroid carcinoma. Int J Endocrinol 2013; 2013: 809382.

32. Ito Y, Miyauchi A. Appropriate treatment for asymptomatic papillary microcarcinoma of the thyroid. Expert Opin Pharmacother 2007; 8: 3205-15.

33. Yu XM, Lloyd R, Chen H. Current treatment of papillary thyroid microcarcinoma. Adv Surg 2012; 46: 191-203.

34. Renshaw AA. Sensitivity of fine-needle aspiration for papillary carcinoma of the thyroid correlates with tumor size. Diagn Cytopathol 2011; 39: 471-4. 